\title{
电子定域化函数的含义与函数形式
}

\author{
卢 天 陈飞武* \\ (北京科技大学化学与生物工程学院, 北京 100083)
}

\begin{abstract}
摘要: 电子定域化函数(ELF) 是研究电子结构的重要工具. 本文介绍了电子定域性的概念, 从电子对密度和动 能密度两个角度详细讨论了 ELF 的物理意义与其函数形式的联系, 并将 ELF 推广到自旋极化形式. 通过实例分 析, 指出了参考项在 ELF 中起到了关键性作用. 对两种自旋极化形式的 ELF 的比较发现: CheckDen 和 TopMoD 程序中使用的形式并不合理, 明显低估了单电子区域的定域性. 最后指出了一些文献由于对 ELF 函数 的错误理解而在引用时出现的错误.
\end{abstract}

关键词：电子定域化函数；动能密度；费米穴；自旋极化；量子化学 中图分类号: 0641

\section{Meaning and Functional Form of the Electron Localization Function}

\author{
LU Tian CHEN Fei-Wu* \\ (School of Chemical and Biological Engineering, University of Science and Technology Beijing, Beijing 100083, P. R. China)
}

\begin{abstract}
The electron localization function (ELF) is an important tool to study electronic structure. In this article, the concept of electron localization is introduced and the overlap between the physical meaning and functional form of the ELF is discussed in detail from two points of view: electron pair density and kinetic energy density. Additionally, we extend the ELF to a generalized spin-polarized form. From case studies we found that the reference term plays an important role in the ELF. A comparison between the two spinpolarized forms of ELF shows that the form used in the CheckDen and TopMoD programs is unreasonable as they obviously underestimate the degree of localization in the single-electron region. Finally, we point out some mistakes in the literature because of a misunderstanding of the ELF.
\end{abstract}

Key Words: Electron localization function; Kinetic energy density; Fermi hole; Spin-polarization; Quantum chemistry

\section{1 引言}

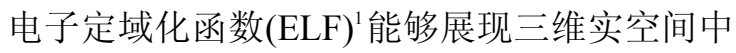
不同位置的电子定域程度, 它易于计算和进行图形 分析, 是量子化学领域中研究电子结构特征的重要 工具. 目前已被广泛应用于各种体系的研究, 如有 机和无机小分子、 ${ }^{2}$ 晶体、 ${ }^{3,4}$ 、固体表面、 ${ }^{5}$ 配位化合 物、 ${ }^{6}$ 团簇 ${ }^{7}$ 和二维量子点. ${ }^{8}$ ELF 也被用于研究不同 问题, 如原子壳层结构、 ${ }^{9}$ 芳香性、10,11 电荷转移键、12
氢键、 ${ }^{13}$ 金属键、 ${ }^{14}$ 多中心键 ${ }^{7,15}$ 和反应机理. ${ }^{16-19}$ ELF 的含时广义化形式 TDELF ${ }^{20}$ 还被用于研究在外场和 散射过程中化学键的动态变化. ELF与分子中的原 子(AIM)理论 ${ }^{21}$ 的拓扑分析方法相互结合, ${ }^{22,23}$ 不仅展 现出更丰富的信息, 也使结果更为清晰, 并上升到 定量高度.

本文主要目的在于理清 ELF 的物理意义与函 数形式之间的联系. 不同研究者对 ELF 的本质提出

Received: August 10, 2011; Revised: September 27, 2011; Published on Web: October 10, 2011.

"Corresponding author. Email: chenfeiwu@ustb.edu.cn; Tel: +86-10-62332689.

The project was supported by the National Natural Science Foundation of China (20773011).

国家自然科学基金(20773011)资助项目

(C) Editorial office of Acta Physico-Chimica Sinica 
了不同的物理解释. 由于在相应讨论时使用的 ELF 形式有所不同, 而且均没有明确指出用于自旋极化 体系时的形式, 导致后续很多研究者没有透彻理解 ELF 的含义与函数形式之间的关系, 而错误地引用 了ELF. 另外 ELF 中参考项引入的意义尚未被着重 讨论过, 其存在的必要性是值得进行分析的. 本文 首先概述电子的定域性与费米穴之间的联系, 然后 从电子对密度和动能密度的角度依次对 ELF 的形 式进行详细讨论, 同时将 ELF 推广到自旋极化形 式. 通过实例, 文中将讨论 ELF 的参考项引入的意 义, 并分析本文提出和已经被一些文献和程序使用 的自旋极化形式之间的差异. 最后指出一些文献中 对 ELF 函数形式引用的错误, 以引起研究者的重视.

\section{2 电子的定域性与费米穴}

在一个局部的三维空间内, 电子的定域性越 高, 说明域内的电子被限制在这个区域内的程度越 高; 域的定域性越低就表明域内的电子越容易离域 出去. 当定域性达到理论最大值时, 则表明电子的 运动完全被限制在域内而不与外部电子交换, 成为 可分辨粒子, 域内的电子数和电子对数也因此是确 定的. ${ }^{21}$ Bader 等 ${ }^{21,24}$ 发现域的定域性与电子的费米穴 有密切的联系. 费米穴可以由费米穴函数 $h$ 来描述, 其表达式为 25,26

$$
h^{\sigma \sigma}\left(\boldsymbol{r}_{1}, \boldsymbol{r}_{2}\right)=\rho_{2, \text { cond }}^{\sigma \sigma}\left(\boldsymbol{r}_{1}, \boldsymbol{r}_{2}\right)-\rho_{\sigma}\left(\boldsymbol{r}_{2}\right)
$$

其中 $\sigma$ 是自旋标号, 为 $\alpha$ 或 $\beta . \boldsymbol{r}$ 是空间坐标. $\rho_{\sigma}(\boldsymbol{r})$ 代表 一个 $\sigma$ 电子出现在 $\boldsymbol{r}$ 处的概率密度. $\rho_{2, \text { cond }}^{\sigma \sigma}\left(\boldsymbol{r}_{1}, \boldsymbol{r}_{2}\right)$ 是 $\sigma$ 电 子间的条件电子对概率密度, 表示已知一个 $\sigma$ 电子 出现在 $\boldsymbol{r}_{1}$ 时另一个 $\sigma$ 电子出现在 $\boldsymbol{r}_{2}$ 时的概率密度. 当 $\rho_{2, \text { ond }}^{\sigma \sigma}\left(\boldsymbol{r}_{1}, \boldsymbol{r}_{2}\right)$ 为 $\rho_{\sigma}\left(\boldsymbol{r}_{2}\right)$ 时, 表示两个电子出现概率没有相 关性, 这时 $h^{\sigma \sigma}\left(\boldsymbol{r}_{1}, \boldsymbol{r}_{2}\right)$ 为零. 实际上, 这只是一种极限 情况. 由于电子之间的 Pauli 互斥和 Coulomb 互斥作 用, $\rho_{2, \text { cond }}^{\sigma \sigma}\left(\boldsymbol{r}_{1}, \boldsymbol{r}_{2}\right)$ 总是小于 $\rho_{\sigma}\left(\boldsymbol{r}_{2}\right)$. 当 $\rho_{2, \text { cond }}^{\sigma \sigma}\left(\boldsymbol{r}_{1}, \boldsymbol{r}_{2}\right)$ 为零时, 表明在一个 $\sigma$ 电子周围没有其他电子, 这时 $h^{\sigma \sigma}\left(\boldsymbol{r}_{1}, \boldsymbol{r}_{2}\right)$ 为 $-\rho_{\sigma}\left(\boldsymbol{r}_{2}\right)$. 这其实也是一种极限情况. 为了研究电子 的定域性, 可以在一个局部空间 $\Omega$ 内对 $h^{\sigma \sigma}\left(\boldsymbol{r}_{1}, \boldsymbol{r}_{2}\right)$ 做积 分, 即

$$
F^{\sigma}(\Omega, \Omega)=\iint_{\Omega \Omega} h^{\sigma \sigma}\left(\boldsymbol{r}_{1}, \boldsymbol{r}_{2}\right) \rho_{\sigma}\left(\boldsymbol{r}_{1}\right) \mathrm{d} \boldsymbol{r}_{1} \mathrm{~d} \boldsymbol{r}_{2}
$$

在该域内 $\sigma$ 电子数目为 $\bar{N}_{\Omega}^{\sigma}=\int_{\Omega} \rho_{\sigma}(\boldsymbol{r}) \mathrm{d} \boldsymbol{r}$. 可以证明, 如 果 $\bar{N}_{\Omega}^{\sigma}$ 个电子完全定域在 $\Omega$ 内, 则 $F^{\sigma}\left(\Omega, \Omega\right.$ 等于 $\bar{N}_{\Omega}^{\sigma}$ 的 负值, 并且这 $\bar{N}_{\Omega}^{\sigma}$ 个电子的费米穴分布一定会全部集
中在 $\Omega$ 内..$^{24}$ 通过考察 $F^{\sigma}(\Omega, \Omega)$ 是否接近 $\bar{N}_{\Omega}^{\sigma}$ 的负值, 可以衡量 $\Omega$ 内 $\sigma$ 电子的定域性高低. 在原子内部壳 层、共价键、孤对电子区域电子具有较高的定域性, 这也说明域内费米穴较大, 相同自旋电子很难接 近. 注意虽然衡量域的定域程度的指标, 即定域化 指数 ${ }^{27}$ 也受到库仑穴的影响, 但是在研究相同自旋 电子对时, 库仑穴起到的影响明显小于费米穴, 故 研究定域性问题时一般可以只考虑费米穴.

费米穴是六维函数, 难以从图形上直观分析, 而通过式(2)研究电子定域性则涉及到 $\Omega$ 的位置和形 状的调整, 不便于实际应用. Bader ${ }^{21}$ 发现电子密度的 拉普拉斯函数具有较大负值的区域, 即电子聚集的 区域内费米穴较大, 因此可以用拉普拉斯函数研究 不同区域的定域性. 然而拉普拉斯函数与电子定域 性之间的理论联系不很紧密, 而且拉普拉斯函数对 电子定域性的表现能力在一些情况下较弱, 如无法 完全展现出序数大于 40 的重原子体系的壳层结 构. ${ }^{28,29}$ 另外, 函数数值范围过大, 没有上限和下限, 因此不便于图形分析, 所以仍需要更好地描述电子 定域性的三维空间函数.

\section{3 电子定域化函数的原始定义}

针对上述问题, Becke 等 ${ }^{1}$ 提出了 ELF, 使得电子 定域性问题可以十分容易地考察. 他们对 HartreeFock 波函数的 $\rho_{2, \text { ond }}^{\sigma \sigma}$ 做球平均化, 然后进行泰勒展 开 $^{30}$

$$
\rho_{2, \text { cond }}^{\sigma \sigma}(\boldsymbol{r}, s)=\frac{1}{3}\left[\sum_{i \in \sigma}^{\mathrm{occ}}\left|\nabla \psi_{i}(\boldsymbol{r})\right|^{2}-\frac{1}{4} \frac{\left|\nabla \rho_{\sigma}(\boldsymbol{r})\right|^{2}}{\rho_{\sigma}(\boldsymbol{r})}\right] s^{2}+\ldots
$$

其中 $\boldsymbol{r}$ 是参考点位置, $s$ 是球半径, $\psi$ 是自旋轨道(下 同), occ 上标代表加和只包括占据轨道. 由于零阶 项(独立于 $s$ ) 对应同时在 $\boldsymbol{r}$ 找到两个 $\sigma$ 电子的概率密 度, 根据 Pauli 互斥原理其值为 0 ; 而一阶项依赖于 费米穴在 $\boldsymbol{r}$ 的梯度, 此处是费米穴极大点, 所以一 阶项也消失, 故式(3)中只含二阶项和高阶项. 式(3) 中方括号内的项对应 $\rho_{2, \text { ond }}^{\sigma \sigma}$ 在 $\boldsymbol{r}$ 的曲率, 下文记做 $D_{\sigma}$. 假设 $\rho_{2, \text { cond }}^{\sigma \sigma}$ 可以被二阶项所精确描述, 那么 $D_{\sigma}$ 可以 被理解为一个 $\sigma$ 电子出现在 $\boldsymbol{r}$ 时, 以其为中心的半径 为 $\sqrt{3}$ 的球壳上另一个 $\sigma$ 电子出现的概率密度. 由于

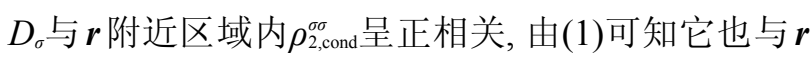
附近 $\sigma$ 费米穴函数呈正相关, 所以实空间函数 $D_{\sigma}$ 可 以用于衡量空间各处电子定域性, 其值越大则 $\boldsymbol{r}$ 处 电子定域性越低, 反之亦然. 
然而 $D_{\sigma}$ 值越大对应定域性越小的行为与习惯 相反, 且没有确定的数值区间, 为此 Becke 等 ${ }^{1}$ 提出 了如下形式的 ELF

$$
\mathrm{ELF}^{\alpha}=\frac{1}{1+\chi_{\sigma}{ }^{2}}
$$

其中

$$
\chi_{\sigma}=D_{\sigma} / D_{\sigma}^{0}
$$

上式中, $D_{\sigma}^{0}$ 是与当前位置具有相同密度的非相互 作用均匀电子气模型下的 $D_{\sigma}$. 在这个模型下, 电子 密度梯度处处为 0 , 所以 $D_{\sigma}^{0}$ 只有 $D_{\sigma}$ 的第一项, 即动 能密度的 2 倍. 而这个模型下的动能密度(轨道单 占据情况时的 Thomas-Fermi 动能密度, 见后文式 (13)) 为 $(3 / 10)\left(6 \pi^{2}\right)^{2 / 3} \rho_{\sigma}^{5 / 3}$, 故

$$
D_{\sigma}^{0}=(3 / 5)\left(6 \pi^{2}\right)^{2 / 3} \rho_{\sigma}^{5 / 3}
$$

式(4)对 $\chi_{\sigma}$ 进行的变换目的是让 ELF 的值域在 $[0,1]$ 内, 从而便于分析, 且让 ELF 值越大时对应越强的 定域性, 使之与常规习惯一致. 由于这个数学变换 并没有明确的物理意义, 故变换方式并不唯一, 有 可能寻找到更好的变换方式使 ELF 有更好的分辨 定域性的能力. 由于 $D_{\sigma}^{0}$ 被作为参考项引入 $E L F$, 所以 $E L F$ 描述的是相对定域性, 当 $D_{\sigma}=D_{\sigma}^{0}$, 即 $E L F=0.5$ 时, 表明当前位置定域性与均匀电子气情况是一致的.

原始的 ELF 只考虑了体系只有一种自旋电子 存在下的定域性问题, 并没有定义当体系同时存在 $\alpha$ 和 $\beta$ 电子时的形式, 但是不难进行扩展. 一个电子 出现在 $\boldsymbol{r}_{1}$ 时, 在 $\boldsymbol{r}_{2}$ 出现相同自旋电子的概率密度, 可 以写为在 $\boldsymbol{r}_{1}$ 出现 $\alpha$ 电子时在 $\boldsymbol{r}_{2}$ 也出现 $\alpha$ 电子的概率密 度以及在 $\boldsymbol{r}_{1}$ 处出现 $\beta$ 电子时在 $\boldsymbol{r}_{2}$ 也出现 $\beta$ 电子的概率 密度的和. 所以, 广义的 ELF 应写为

$$
\mathrm{ELF}=\frac{1}{1+\chi^{2}}
$$

其中

$$
\begin{aligned}
\chi & =\frac{(1 / 2)\left(D_{\alpha}+D_{\beta}\right)}{(1 / 2)\left(D_{\alpha}^{0}+D_{\beta}^{0}\right)} \\
& =\frac{\frac{1}{2} \sum_{i}^{\text {occ }}\left|\nabla \psi_{i}\right|^{2}-\frac{1}{8}\left[\frac{\left|\nabla \rho_{\alpha}\right|^{2}}{\rho_{\alpha}}+\frac{\left|\nabla \rho_{\beta}\right|^{2}}{\rho_{\beta}}\right]}{(3 / 10)\left(6 \pi^{2}\right)^{2 / 3}\left(\rho_{\alpha}{ }^{5 / 3}+\rho_{\beta}{ }^{5 / 3}\right)}
\end{aligned}
$$

上式在分子分母上都乘以 $1 / 2$ 是为了方便与下文 Savin 定义的形式相对照.

对于闭壳层体系, 由于 $\rho_{a}=\rho_{\beta}=(1 / 2) \rho$, 故

$$
\begin{aligned}
& \rho_{\sigma}^{5 / 3}=\rho_{\beta}^{5 / 3}=(1 / 2)(1 / 2)^{2 / 3} \rho^{5 / 3} \\
& \frac{\left|\nabla \rho_{\alpha}\right|^{2}}{\rho_{\alpha}}=\frac{\left|\nabla \rho_{\beta}\right|^{2}}{\rho_{\beta}}=\frac{1}{2} \frac{|\nabla \rho|^{2}}{\rho}
\end{aligned}
$$

因此, 式(8)可改写为

$$
\chi=\frac{\frac{1}{2} \sum_{i}^{\text {occ }}\left|\nabla \psi_{i}\right|^{2}-\frac{1}{8} \frac{|\nabla \rho|^{2}}{\rho}}{(3 / 10)\left(3 \pi^{2}\right)^{2 / 3} \rho^{5 / 3}}
$$

\section{4 动能角度对电子定域化函数的定义}

Becke 基于电子对密度定义的 ELF 只适合 Hartree-Fock 波函数, 尽管 Kohn-Sham 密度泛函 (KS-DFT)波函数同样具有单行列式波函数形式, 但 是 KS-DFT波函数引入的意义在于获得真实的密度 分布以及获得较精确的动能, 而不是像 HF 波函数 那样作为真实波函数的近似, 所以 Becke 的 ELF 从 物理意义的角度上不能用在 KS-DFT 波函数上. Savin 等 ${ }^{4,22}$ 对 ELF 从动能角度进行了新的定义, 使 得 ELF 的适用范围扩展到 KS-DFT 波函数. 这种定 义与原始形式差别仅在于 $\chi$ 项, 写为

$$
\chi=\frac{\tau_{\mathrm{r}}-\tau_{\mathrm{w}}}{\tau_{\mathrm{h}}}
$$

其中

$$
\begin{aligned}
& \tau_{\mathrm{r}}=(1 / 2) \sum_{i}^{o c c}\left|\nabla \psi_{i}\right|^{2}=(1 / 8) \sum_{i}\left|\nabla \rho_{i}\right|^{2} / \rho_{i} \\
& \tau_{\mathrm{w}}=(1 / 8)|\nabla \rho|^{2} / \rho, \quad \tau_{\mathrm{h}}=(3 / 10)\left(3 \pi^{2}\right)^{2 / 3} \rho^{5 / 3}
\end{aligned}
$$

式中, $\tau_{\mathrm{r}}$ 代表当前体系的正定动能密度, ${ }^{26}$ 可视为动 能密度的精确值; $\tau_{\mathrm{h}}$ 是 Thomas-Fermi (TF) 非相互作 用均匀电子气模型的动能密度, 其中常数项被称为 $\mathrm{TF}$ 常数, 其倒数为 $0.34828 ; \tau_{\mathrm{w}}$ 是 Von Weizsäcker $(\mathrm{VW})$ 动能密度. ${ }^{31}$ 由式(11)可见, 当某个位置仅有一 条轨道出现时, $\mathrm{VW}$ 动能密度与精确动能密度相等. 对于非相互作用的玻色子体系, 由于没有 Pauli 不相 容原理的限制, 全部粒子可以占据同一条轨道, 故 VW 动能密度精确描述了非相互作用粒子体系无 Pauli 互斥作用时的动能密度. $\tau_{\mathrm{r}}-\tau_{\mathrm{w}}$ 总为正值, ${ }^{32}$ 它被 称为 Pauli动能密度, 是 Pauli 互斥导致的超额动能. ${ }^{33}$ Pauli 动能密度越小的区域电子受相同自旋电子影 响越小, 因此定域性越大. 由于式(10)中存在参考项 $\tau_{\mathrm{h}}$, 所以应认为 Savin 的 ELF 衡量的也是相对定域性 而不是绝对定域性. 值得一提的是, 如果将 $\tau_{\mathrm{r}}$ 项写为 自然轨道的形式, 这种基于动能密度定义的 ELF也 可以用于后 $\mathrm{HF}$ 波函数.

与 Becke 的定义方式相反, Savin 的定义未明确 考虑自旋, 只适合用于闭壳层体系. 将式(9)与式(10)、 (11)进行比较, 可见两种定义用于闭壳层体系时形式 是一致的. 对于自旋极化体系, 即 $\rho_{\alpha} \neq \rho_{\beta}$ 的情况, Savin 
的 ELF 需要进行推广. 根据 Oliver 和 Perdew ${ }^{34}$ 对非 相互作用体系的自旋极化形式动能泛函的定义

$$
T\left[\rho_{a}, \rho_{\beta}\right]=(1 / 2) T\left[2 \rho_{a}\right]+(1 / 2) T\left[2 \rho_{\beta}\right]
$$

自旋极化形式的 $\mathrm{TF}$ 和 VW 动能密度应写为

$$
\begin{gathered}
\tau_{\mathrm{h}}=(3 / 10)\left(6 \pi^{2}\right)^{2 / 3}\left(\rho_{\alpha}^{5 / 3}+\rho_{\beta}{ }^{5 / 3}\right) \\
\tau_{\mathrm{w}}=\frac{1}{8} \frac{\left|\nabla \rho_{\alpha}\right|^{2}}{\rho_{\alpha}}+\frac{1}{8} \frac{\left|\nabla \rho_{\beta}\right|^{2}}{\rho_{\beta}}
\end{gathered}
$$

将式(13)代入式(10), 并与式(8)进行对比, 发现从电子 对密度和动能密度角度得到的自旋极化形式的 ELF 也 是完全一致的.

Bader 等 ${ }^{35}$ 将动能密度、定域化轨道和费米穴概 念相结合, 给 ELF 的本质做了另一种解释. 在单行 列式波函数下, 式(2)可以更明确地写为

$$
F(\Omega, \Omega)=-\sum_{i \in \sigma}^{\mathrm{occ}} \sum_{j \in \sigma}^{\mathrm{occ}} S_{i, j}^{2}(\Omega)
$$

其中 $S_{i j}(\Omega)$ 代表 $i v j$ 自旋轨道在 $\Omega$ 域内的重叠积分. 只 有轨道能够分立地定域化的时候 $F(\Omega, \Omega)$ 才能达到 极限值, 即 $\bar{N}_{\Omega}^{\sigma}$ 的负值, 这时出现完全定域化域. ${ }^{21}$ 所谓 分立定域化是指某轨道涉及的空间范围构成独立的 域, 在其中此轨道不与其它轨道发生重叠. 容易理 解, 正是由于描述域内电子运动的轨道与域外部的 轨道间彼此分立, 才使域内电子不会离域到域外. 由 于在域内 $\tau_{\mathrm{r}}$ 与 $\tau_{\mathrm{w}}$ 精确相等, 因此 ELF会达到应有的最 大值 1 . 虽然分立的定域化对于非孤立体系是无法满 足的, 但是在孤对电子区域以及共价键区域内一般 只由一个定域化轨道所主导, 因此 $\tau_{\mathrm{r}}-\tau_{\mathrm{w}}$ 会较小, 使 ELF 能正确地表现出这些区域的高定域性特征.

值得一提的是在电子远离分子骨架时, 其整体 密度往往只由一条轨道的密度所描述..$^{33}$ 此时 VW动 能密度会十分接近精确动能密度, 导致 $\tau_{\mathrm{r}}-\tau_{\mathrm{w}}$ 项经常 比 $\tau_{\mathrm{h}}$ 项更快收玫至 0 , 使 ELF 值为 1 而显示出虚假的 高定域性. 我们建议在 $\chi$ 项的分子上加入一个很小 值, 如 0.00001 . 在分子内部区域由于动能密度较大, 这个处理不会影响 ELF 的行为; 而在逐渐远离分子 而动能密度趋近于 0 的过程中, 这个处理可保证 ELF 正确收敛到 0 .

\section{5 电子定域化函数中参考项的意义}

从前面的讨论中已看到, 不同的研究者对 ELF 的本质从三个角度进行了清晰的解释, 然而这些解 释都只关注 $\chi$ 项的分子, 却没有重视 $\chi$ 项的分母, 分母 项在 ELF 中存在的必要性是值得探究的. 这里我们 将以氧化氢分子为实例考察分母项引入 ELF 的意
义, 以 Savin对动能密度的解释为切入点进行探讨. 下 文中几何优化和波函数计算使用 Gaussian 03 程序, ${ }^{36}$ 理论方法为 $\mathrm{B} 3 \mathrm{LYP},{ }^{37}$ 基组使用 $6-311 \mathrm{G}(2 d f, p),{ }^{38,39}$ ELF 的计算使用我们开发的 Multiwfn 2.0 程序. ${ }^{40}$ 如 上文所提到的, $\tau_{\mathrm{r}}-\tau_{\mathrm{w}}$ 项都加入 0.00001 以避免 ELF 在远离分子处出现虚假高定域性.

图 1 显示了 $\mathrm{HCN}$ 轴线上 Pauli 动能密度与 $\mathrm{TF}$ 动 能密度的分布. TF 动能密度的行为与电子密度完全 一致, 在原子核处存在岐点, 随着远离原子其数值 呈指数型衰减. 按照 Savin 的解释, ELF 能够展现高 定域性的区域是因为在相应区域有较低的 Pauli 动 能密度, 然而从图中可见, Pauli 动能密度的分布类 似于电子密度, 都是在重原子核附近较大而在较远 区域很小, 并没有提供新的信息, $\mathrm{C} \equiv \mathrm{N}$ 共价键和 $\mathrm{N}$ 的孤对电子都没有显现出来. 唯一明显区别是 Pauli 动能密度在重原子的原子核位置存在极小点, 这主 要是由于 Gauss 基函数在原子核处导数为零导致 的. 只有当 Pauli 动能密度和 $\mathrm{TF}$ 动能密度相互比较 时电子定域性的信息才能得到展现, 可以看到在 ELF 显示的高定域性区域, 即氢原子核附近、C 和 $N$ 原子核附近、成键及孤对电子区域Pauli 密度都要小 于 TF 动能密度, 将这两种动能密度相除得到的 $\chi$ 项 如图 2 虚线所示, 不同区域定域性的高低已经变得 十分明显. 将 $\chi$ 项按照式(7)变换就得到了 ELF, 从图 上看这个变换过程只是使定域性在分析时更方便, 却并不是绝对必要的.

上述分析表明, 仅凭 Pauli 动能项是无法令 ELF 发挥分辨不同区域定域性高低的能力的. Pauli 动能项虽然包含了电子定域性的信息, 但是其数值 很大程度由电子密度所主导, 为了使定域性信息得

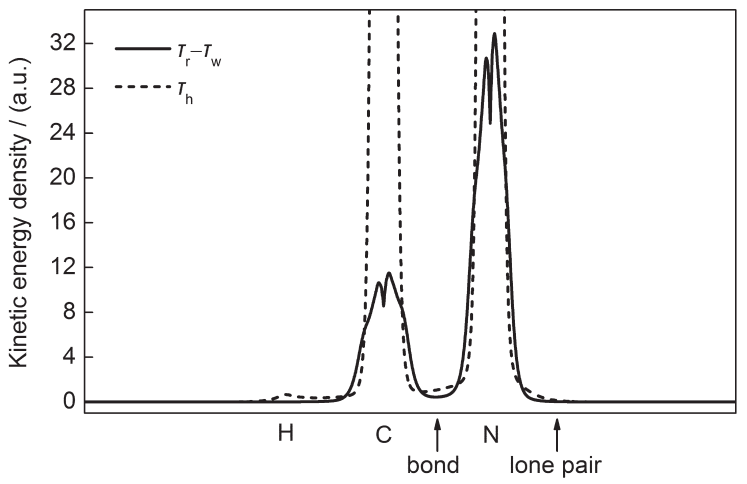

图 $1 \mathrm{HCN}$ 的轴线方向上 Pauli 动能密度(实线)与 TF 动能密度(虚线)

Fig.1 Pauli kinetic energy density (solid line) and TF kinetic energy density (dashed line) on the axis of $\mathrm{HCN}$ The positions of nuclei are marked by corresponding letters. 


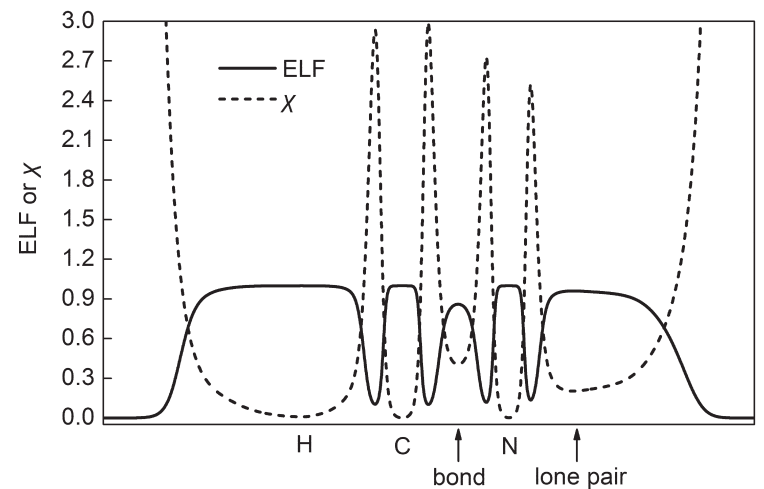

图 $2 \mathrm{HCN}$ 的轴线方向上 $\mathrm{ELF}$ 数值(实线) 与 $\chi$ 项数值(虚线)

Fig.2 The value of ELF (solid line) and $\chi$ term (dashed line) on the axis of $\mathrm{HCN}$

The positions of nuclei are marked by corresponding letters.

到清晰展现, 就必须将 TF 动能密度作为参考项引 入来抵消掉电子密度的主导效应. 可以说 $\chi$ 项是 ELF 的核心, 构成它的 Pauli 动能密度与 TF 动能密 度项缺一不可。

\section{6 两种自旋极化形式的电子定域化函数的 差异}

前文中已经给出了合理的 ELF 自旋极化形式, 即式(8). 然而在一些计算 ELF 的程序中, 如 Check$\mathrm{Den}^{41}$ 和 TopMoD, ${ }^{42}$ 对于自旋极化体系使用的是直 接将 $\rho=\rho_{\alpha}+\rho_{\beta}$ 代入闭壳层 ELF 得到的形式

$$
\chi=\frac{\frac{1}{2} \sum_{i}^{o c c}\left|\nabla \psi_{i}\right|^{2}-\frac{1}{8} \frac{\left|\nabla \rho_{\alpha}+\nabla \rho_{\beta}\right|^{2}}{\rho_{\alpha}+\rho_{\beta}}}{(3 / 10)\left(3 \pi^{2}\right)^{2 / 3}\left(\rho_{\alpha}+\rho_{\beta}\right)^{5 / 3}}
$$

这种 ELF 自旋极化形式并没有严格的理论依据. 为 了考察使用式(8)与使用式(15)所得结果的差异, 本 文选取了三个自旋极化体系进行比较研究, 即三重 态二氟亚甲基、单重态 1,4-丁烷双自由基和钾原子.

图 3(a,b) 展现了三重态二氟亚甲基的垂面的

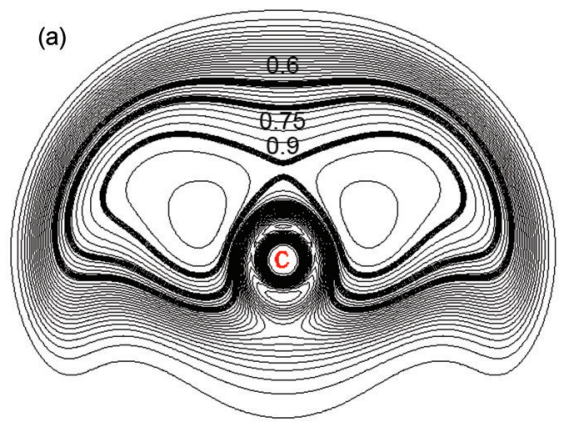

ELF 等值线图, 分别由式(8)和(15)计算得到. 从两幅 图中都可以明显看到碳原子的两个自旋平行的电 子由于Pauli互斥而相互远离, 分别定域在分子平面 的两侧, 显示出较大 ELF 数值. 图 3 也展现出两种 ELF 自旋极化形式在定量上的差异, 由式(8)定义所 显示的未成对电子对应的高定域性区域更大, 0.9 的 等值线包围的范围相较图 3(b) 明显膨胀, 并且 0.96 的等值线也已经出现.

在单重态1,4-丁烷双自由基中, 两个自旋相反 的单电子分别定域在两端碳原子上, 相应位置也具 有较大自旋密度. 从图 4(a)来看, 由式(8)定义的 ELF 很明确地指出了单电子出现的位置, 并且对应的高 定域性区域较大(与碳碳 $\sigma$ 键区域有一定连通), 与期 望的完全一致. 而在图 4(b)中, 单电子对应的高定域 性区域显著缩小, 相应的 0.75 的等值线包围的区域 仅有图 4(a)的约 1/5, 这表明式(15)的定义严重低估 了自由基单电子的定域性, 同时也给图形化分析带 来了一定不便.

图 5 显示了钾原子的径向 ELF 分布. 由于自旋 极化对内层电子基本不产生影响, 所以两种定义显 示出完全相同的内部壳层结构. 而由于自旋密度集 中在价层, 两种定义在描述价层结构上出现分歧, 式(15)对应的结果低估了 $4 s$ 电子的定域性, 并且峰 的位置稍微往外移动, 但它们表现的基本特征仍然 是一致的.

由以上例子可见, 式(8)定义的 ELF 比式(15)的 结果在高自旋极化区域总是有更大的数值, 这一方 面是由于式(8)的分子总小于等于式(15)的, 因为

$$
\begin{aligned}
& \frac{\left|\nabla \rho_{\alpha}\right|^{2}}{\rho_{\alpha}}+\frac{\left|\nabla \rho_{\beta}\right|^{2}}{\rho_{\beta}}-\frac{\left|\nabla \rho_{\alpha}+\nabla \rho_{\beta}\right|^{2}}{\rho_{\alpha}+\rho_{\beta}}= \\
& \frac{\left(\rho_{\beta} \nabla \rho_{\alpha}-\rho_{\alpha} \nabla \rho_{\beta}\right)^{2}}{\rho_{\alpha} \rho_{\beta}\left(\rho_{\alpha}+\rho_{\beta}\right)} \geq 0
\end{aligned}
$$

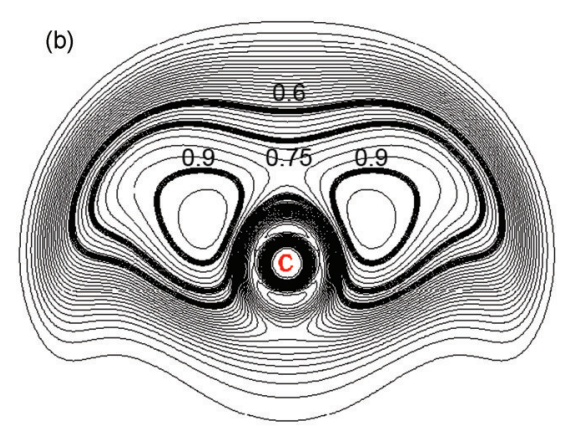

图 3 三重态二氟亚甲基的包含碳原子的垂直平面上的 ELF 等值线图

Fig.3 ELF contour line map of triplet difluoro-methylene on the vertical plane containing carbon atom

For the convenience of comparison, several contour lines are bolded. The values in (a) and (b) are computed according to Eq.(8) and Eq.(15), respectively. The isovalues start from 0 with stepsize of 0.03 , similarly hereinafter. 

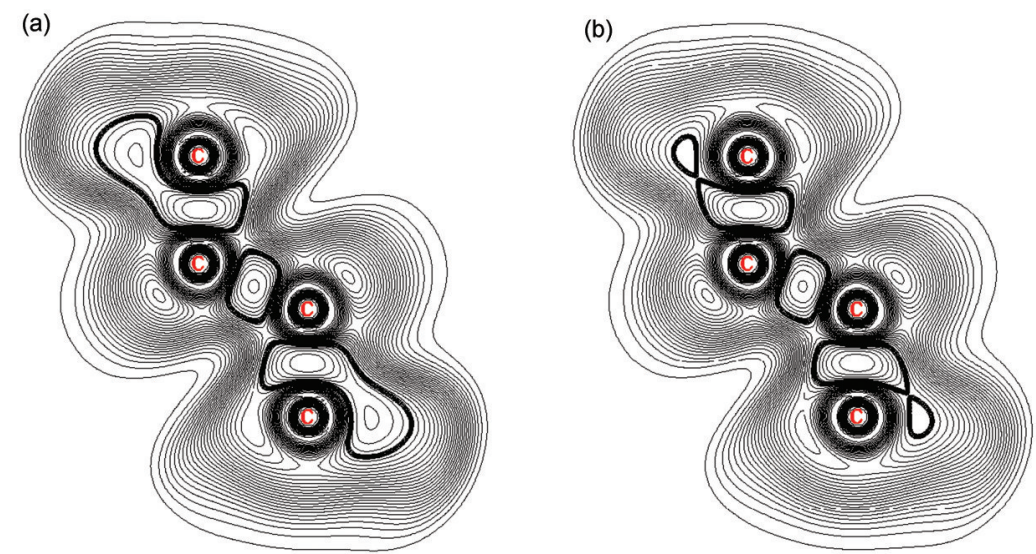

图 4 单重态 1,4-丁烷双自由基在 $C_{2 h}$ 对称平面上的 ELF 等值线图

Fig.4 ELF contour line map on the $C_{2 h}$ symmetrical plane of singlet 1,4-butane biradical

(a) and (b) correspond to the ELF defined by Eq.(8) and Eq.(15), respectively. The contour line with value of 0.75 is bolded.

另一方面通过数值分析可以验证 $2^{2 / 3}\left(\rho_{\alpha}^{5 / 3}+\rho_{\beta}^{5 / 3}\right)$ 值总 大于 $\left(\rho_{\alpha}+\rho_{\beta}\right)^{5 / 3}$ 值, 并且其差值 $\Delta$ 与自旋密度 $\rho^{\mathrm{s}}$ 呈正相 关, 通过拟合可将关系表示为 $\Delta=0.363 \rho^{\mathrm{s}}$, 所以式(8) 的分母比式(15)的更大. 分子和分母这两个因素共 同导致式(8)比式(15)更小, 故前者对应的 ELF 数值 总是比后者更大, 在单电子定域性越高的区域差异 越显著. 虽然从例子中也看到式(15)可以得到与式 (8)定性一致的结论, 然而由于式(15)的定义缺乏物 理意义, 而且在诸如自由基情况下对电子定域性分 辨能力弱, 所以对于自旋极化体系应使用式(8).

\section{7 文献中对电子定域化函数的错误引用}

我们发现, 在许多引用 ELF 的文献中给出的函 数形式存在错误, 下面列举几个例子.

在文献 ${ }^{2}$ 当中使用的是 ELF 闭壳层形式, $\chi$ 项被 写为

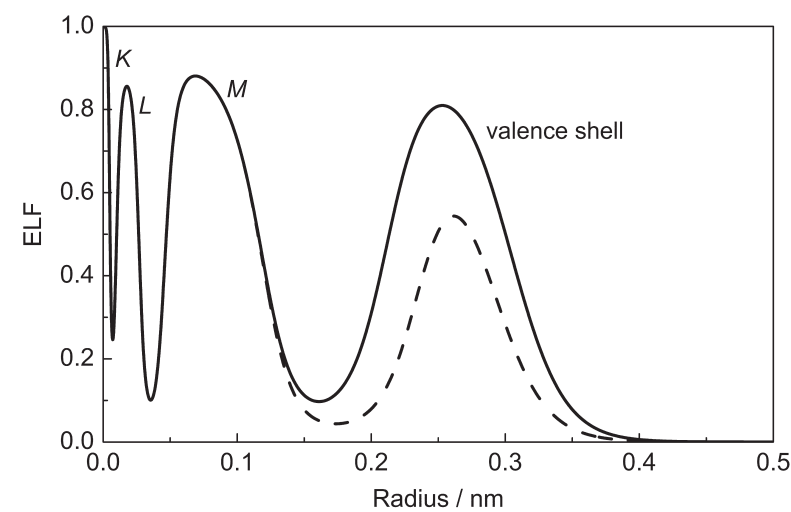

图 5 钾原子的径向 ELF 图

Fig.5 Radial ELF map of potassium atom

Solid line and dashed line correspond to the ELF defined by Eq.(8) and Eq.(15), respectively.

$$
\chi=0.3483 \rho^{-5 / 3}\left[\sum_{i}^{\mathrm{occ}}\left|\nabla \psi_{i}\right|^{2}-(1 / 8)|\nabla \rho|^{2} / \rho\right]
$$

其中 $\psi$ 仍代表自旋轨道. 对照式(10)和(11), 发现在 $\tau_{\mathrm{r}}$ 项中缺少系数 $1 / 2$. 其错误原因可能源于 Becke 在 $\mathrm{ELF}$ 原始文献 ${ }^{1}$ 中省略了系数, 而将 $\left.\sum_{i}\left|\nabla \psi_{i}\right|^{2}\right|^{2}$ 尔为正 定动能密度, 由于 $\tau_{\mathrm{w}}$ 与 $\tau_{\mathrm{h}}$ 项也都省略了 $1 / 2$, 求比值时 缺少的项相抵消, 所以原文中形式正确, 而在文献 ${ }^{2}$ 中却只有 $\tau_{\mathrm{r}}$ 中省略了 $1 / 2$, 所以形式是错误的.

在文献 ${ }^{43}$ 当中通过电子对概率密度的角度对 ELF 进行了引用, $\chi_{\circ}$ 被写为

$$
\chi_{\sigma}=0.3483 \rho_{\sigma}{ }^{-5 / 3}\left[\sum_{i \in \sigma}^{o c c}\left|\nabla \psi_{i}\right|^{2}-(1 / 8)\left|\nabla \rho_{\sigma}\right|^{2} / \rho_{\sigma}\right]
$$

对照正确的条件电子对概率密度在参考点处的曲 率 $\left(D_{\sigma}\right)$, 即式(3)的方括号内的项, 上式中的 $1 / 8$ 应当 为 $1 / 4$. 另外, 文中错误地将只含单一的自旋电子时 $\chi_{\sigma}$ 的分母项, 即式(6), 错误地写作闭壳层ELF 表达式 (9) 的分母项, 所以式中的 0.3483 应替换为 $1 /[(3 / 5)$ $\left.\left(6 \pi^{2}\right)^{2 / 3}\right]=0.1097$.

在文献 ${ }^{42}$ 的式(6)、(7)当中, 从动能密度角度对 单一自旋形式的 ELF 进行了引用, 等价表述为

$$
\chi_{\sigma}=\frac{T_{\mathrm{s}}[\rho]-(1 / 4)\left|\nabla \rho_{\sigma}\right|^{2} / \rho_{\sigma}}{(3 / 10)\left(3 \pi^{2}\right)^{2 / 3} \rho^{5 / 3}}
$$

其中精确动能项 $T_{\mathrm{s}}$ 的变量 $\rho$ 以及分母中的 $\rho$ 都缺失了 自旋标号, 如果被替换为 $\rho_{\sigma}$, 对比本文式(13)就会发 现两个问题, 一个是 VW 动能密度项中的系数应为 $1 / 8$ 而非 $1 / 4$, 另外 $\mathrm{TF}$ 动能密度的自旋极化形式中的 $6 \pi^{2}$ 被错误地写为了闭壳层形式的 $3 \pi^{2}$.

由上可见, 文献中引用 ELF 时, 在 $\chi$ 的分母中常 
常将自旋极化和非自旋极化形式相混淆, 而在分子 中经常缺失系数. 为了避免引用时的错误, 应注意 理解ELF 各项的含义并分清不同情况下的形式.

\section{8 总 结}

介绍了电子定域性的基本概念, 详细讨论了 ELF 的三种物理解释, 分别从电子对密度和动能密度 两个角度将ELF扩展到自旋极化形式, 得到了完全相 同的形式. 通过研究 ELF 及组成它的各个成分在 $\mathrm{HCN}$ 分子中的分布发现, 参考项在 ELF 中起着至关 重要的作用, 缺失了参考项的 ELF 将不能分辨不同区 域电子定域性的高低, 然而参考项的意义却往往没 有得到重视. 一些程序中, 如 CheckDen 和 TopMoD, 使用的 ELF 自旋极化形式是直接将闭壳层形式中 的总密度改写为 $\alpha$ 和 $\beta$ 密度加和得到的. 通过本文的 分析, 发现虽然这种形式也可以得到定性合理的结 果, 但是在自旋密度较大区域低估了电子定域性, 并且由于这种形式没有严格的物理意义, 是不建议 使用的. 通过比较本文和一些文献给出的 ELF 的形 式, 发现有些文献使用的形式有误, 为避免错误的 出现, 需要研究者注意认清 ELF 的物理意义与函数 形式之间的关系.

\section{References}

(1) Becke, A. D.; Edgecombe, K. E. J. Chem. Phys. 1990, 92, 5397.

(2) Savin, A.; Becke, A. D.; Flad, J.; Nesper, R.; Preuss, H.; von Schnering, H. G. Angew. Chem. Int. Edit. 1991, 30, 409.

(3) Grin, Y.; Wagner, F. R.; Armbrüster, M.; Kohout, M.; LeitheJasper, A.; Schwarz, U.; Wedig, U.; von Schnering, H. G. J. Solid State Chem. 2006, 179, 1707.

(4) Savin, A.; Jepsen, O.; Flad, J.; Andersen, O. K.; Preuss, H.; von Schnering, H. G. Angew. Chem. Int. Edit. Engl. 1992, 31, 187.

(5) Gomes, J. R. B.; Illas, F.; Silvi, B. Chem. Phys. Lett. 2004, 388, 132.

(6) Kohout, M.; Wagner, F. R.; Grin, Y. Theor. Chem. Acc. 2002, $108,150$.

(7) Rousseau, R.; Marx, D. Chem. -Eur. J. 2000, 6, 2982.

(8) Räsänen, E.; Castro, A.; Gross, E. K. U. Phys. Rev. B 2008, 77, 115108.

(9) Kohout, M.; Savin, A. Int. J. Quantum Chem. 1996, 60, 875.

(10) Poater, J.; Duran, M.; Solà, M.; Silvi, B. Chem. Rev. 2005, 105, 3911.

(11) Santos, J. C.; Andres, J.; Aizman, A.; Fuentealba, P. J. Chem. Theory Comput. 2004, 1, 83.

(12) Shaik, S.; Danovich, D.; Silvi, B.; Lauvergnat, D. L.; Hiberty, P. C. Chem. -Eur. J. 2005, 11, 6358.

(13) Grabowski, S. J. Chem. Rev. 2011, 111, 2597.
(14) Llusar, R.; Beltrán, A.; Andrés, J.; Fuster, F.; Silvi, B. J. Phys. Chem. A 2001, 105, 9460.

(15) Molina, J.; Dobado, J. A. Theor. Chem. Acc. 2001, 105, 328.

(16) Berski, S.; Andrés, J.; Silvi, B.; Domingo, L. R. J. Phys. Chem. A 2003, 107, 6014.

(17) Polo, V.; Andres, J.; Berski, S.; Domingo, L. R.; Silvi, B. J. Phys. Chem. A 2008, 112, 7128.

(18) Krokidis, X.; Noury, S.; Silvi, B. J. Phys. Chem. A 1997, 101, 7277.

(19) Chamorro, E. J. Chem. Phys. 2001, 114, 23.

(20) Burnus, T.; Marques, M. A. L.; Gross, E. K. U. Phys. Rev. A 2005, 71, 010501.

(21) Bader, F. W. Atoms in Molecules: A Quantum Theory; Oxford University Press: New York, 1994; pp 332-343.

(22) Silvi, B.; Savin, A. Nature 1994, 371, 683.

(23) The Quantum Theory of Atoms in Molecules—From Solid State to DNA and Drug Design; Matta, C. F., Boyd, R. J. Eds.; WILEY-VCH Verlag GmbH \& Co. KGaA: Weinheim, 2007.

(24) Bader, R. F. W.; Stephens, M. E. J. Am. Chem. Soc. 1975, 97 , 7391.

(25) Koch, W.; Holthausen, M. C. A Chemist's Guide to Density Functional Theory, 2nd ed.; Wiley-VCH Verlag GmbH: Germany, 2001.

(26) McWeeny, R. Methods of Molecular Quantum Mechanics, 2nd ed.; Academic Press: San Diego, 1992.

(27) Fradera, X.; Austen, M. A.; Bader, R. F. W. J. Phys. Chem. A 1998, 103, 304.

(28) Sagar, R. P.; Ku, A. C. T.; Smith, J. V. H.; Simas, A. M. J. Chem. Phys. 1988, 88, 4367.

(29) Shi, Z.; Boyd, R. J. J. Chem. Phys. 1988, 88, 4375.

(30) Becke, A. D. Int. J. Quantum Chem. 1985, 27, 585.

(31) von Weizsäcker, C. F. Z. Phys. 1935, 96, 431.

(32) Tal, Y.; Bader, R. F. W. Int. J. Quantum Chem. 1978, 14, 153.

(33) Lignères, V. L.; Carter, E. A. An Introduction to Orbital-Free Density Functional Theory. In Handbook of Materials Modeling; Yip, S. Ed.; Springer: Netherlands, 2005; pp 137-148.

(34) Oliver, G. L.; Perdew, J. P. Phys. Rev. A 1979, 20, 397.

(35) Bader, R. F. W.; Johnson, S.; Tang, T. H.; Popelier, P. L. A. J. Phys. Chem. 1996, 100, 15398.

(36) Frisch, M. J.; Trucks, G. W.; Schlegel, H. B.; et al. Gaussian 03, Revison E.01; Gaussian Inc.: Wallingford, CT, 2004.

(37) Becke, A. D. J. Chem. Phys. 1993, 98, 1372.

(38) Krishnan, R.; Binkley, J. S.; Seeger, R.; Pople, J. A. J. Chem. Phys. 1980, 72, 650.

(39) Hariharan, P. C.; Pople, J. A. Theor. Chem. Acc. 1973, 28, 213.

(40) Lu, T. Multiwfn, 2.0, 2011. http://Multiwfn.codeplex.com.

(41) Pacios, L. F.; Fernandez, A. J. Mol. Graph. Model. 2009, 28, 102.

(42) Noury, S.; Krokidis, X.; Fuster, F.; Silvi, B. Comput. Chem. 1999, 23, 597.

(43) Burdett, J. K.; McCormick, T. A. J. Phys. Chem. A 1998, 102, 6366. 